\title{
Nickel-Catalyzed Reductive Arylation of Redox Active Esters for the Synthesis of $\alpha$-Aryl Nitriles - Role of a Chlorosilane Additive
}

\author{
Nicholas W. M. Michel§, Racquel K. Edjoc§, Emmanuel Fagbola§, Jonathan M. E. Hughesł, Louis- \\ Charles Campeaut, Sophie A. L. Rousseaux $*^{*}$
}

AUTHOR ADDRESS

§ Department of Chemistry, University of Toronto, Toronto, Ontario M5S 3H6, CANADA
\$Department of Process Research and Development, Merck \& Co. Inc., Rahway, New Jersey 07065, United States
KEYWORDS nickel catalysis, reductive cross-coupling, chlorosilane, $\alpha$-aryl nitrile, arylation, radical, NHP ester

ABSTRACT: A nickel-catalyzed reductive cross-coupling of redox-active $N$-hydroxyphthalimide (NHP) esters and iodoarenes for the synthesis of $\alpha$-aryl nitriles is described. The NHP ester substrate is derived from cyanoacetic acid, which allows for a modular synthesis of substituted $\alpha$-aryl nitriles, an important scaffold in pharmaceutical sciences. Mechanistic studies reveal that decarboxylation of the NHP ester to the reactive radical intermediate is accomplished by a combination of a chlorosilane additive and $\mathrm{Zn}$ dust. The reaction exhibits a broad scope as many functional groups are compatible under the reaction conditions, including complex highly functionalized medicinal agents.

Scheme 1. $\alpha$-Aryl nitrile synthesis via cross-coupling

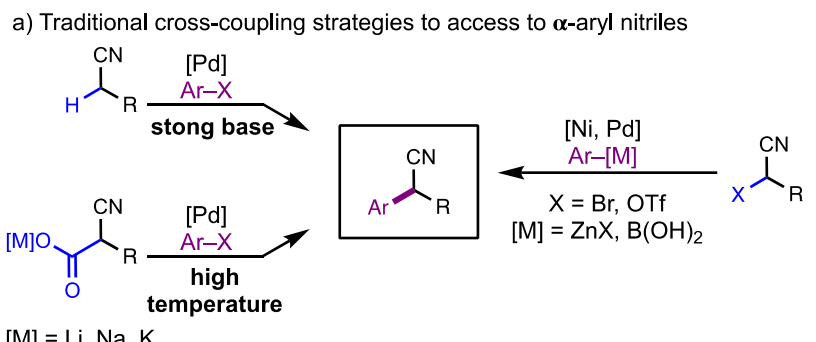

$[\mathrm{M}]=\mathrm{Li}, \mathrm{Na}, \mathrm{K}$

b) Reductive cross-coupling strategies to access to $\boldsymbol{\alpha}$-arylnitrile

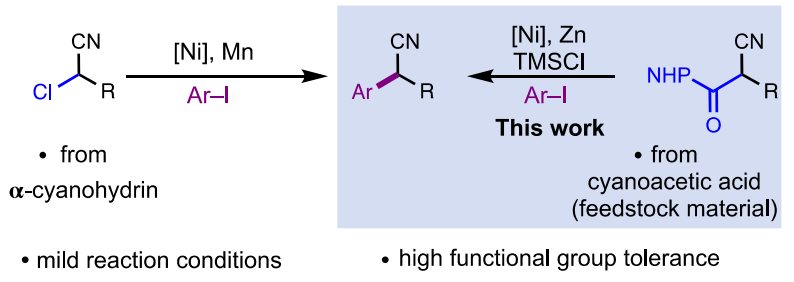

Nickel-catalyzed reductive cross-couplings (RCC) have garnered considerable interest over the past decade since they allow for the construction of $\mathrm{C}-\mathrm{C}$ bonds from two distinct electrophilic reagents. ${ }^{1}$ Compared to traditional crosscoupling reactions, reductive methods tend to avoid the discrete formation of anionic species which often leads to milder reaction conditions that exhibit exceptional functional group tolerance. Thus, reductive cross-coupling reactions that target substrates containing base-sensitive functional groups are highly attractive as they elegantly showcase the advantages of this cross-coupling strategy. One such example that would benefit from this strategy is the synthesis of $\alpha$-aryl nitriles. These scaffolds are of importance to the synthetic community due to their versatile nature as synthetic intermediates and their prevalence in pharmaceutical agents. Thus, considerable efforts have been dedicated to their synthesis. $^{2}$

Traditionally, feedstock chemicals such as aliphatic nitriles ${ }^{3}$ or cyanoacetate salts ${ }^{4}$ can undergo $\alpha$-arylation as a nucleophilic reagent in the presence of an aryl(pseudo)halide coupling partner with a transition-metal catalyst such as Pd (Scheme 1a, left). However, these established $\alpha$-arylation methods require strongly basic reaction conditions and/or high temperatures to generate the nucleophilic coupling partner, which limits both their functional group compatibility and their use in late-stage diversification of complex molecules. In contrast, cross-coupling reactions using $\alpha$ (pseudo)halo nitriles as electrophilic coupling partners address these limitations as the reactions tend to be milder. As such, $\alpha$-(pseudo)halo nitriles have been elegantly employed in traditional $^{5}$ (Scheme 1a, right), and reductive ${ }^{6,7}$ (Scheme $1 \mathrm{~b}$, left) cross-coupling reactions. The main drawback to these methods is that starting material preparation can be undesirable since they are typically synthesized from a cyanohydrin intermediate which requires the use of a toxic cyanide equivalent. As an alternative approach, we envisioned using cyanoacetic acid derived electrophiles in a RCC as these are feedstock starting materials and easily derivatized (Scheme 1b, blue box). Specifically, we envisioned using the redox properties of $N$-hydroxyphthalimide (NHP) esters to generate an $\alpha$-cyano radical that can engage in Ni-catalyzed arylation. ${ }^{8}$

RCC reactions of NHP esters are powerful methods that have been used to prepare a diverse range of $\mathrm{C}-\mathrm{C}$ bonds. ${ }^{9}$ Notably, while there are many examples in the literature describing the functionalization of alkyl substituted NHP ester derivatives, examples of NHP esters bearing additional functional group 
handles in the $\alpha$-position are rare. ${ }^{10}$ Herein we describe the development of a reductive decarboxylative arylation reaction of cyanoacetic-derived NHP esters. Mechanistic studies of the reaction revealed a unique reduction mechanism for the NHP ester substrate and indicated that an $\alpha$-cyano radical intermediate is generated in the combined presence of a chlorosilane additive and $\mathrm{Zn}$ dust. This finding has implications on the field of NHP ester functionalization, as the role of halosilane additives in previous investigations are not fully understood. ${ }^{9 b}$

We began reaction optimization by exploring the RCC of NHP ester 1a and iodoarene 2. Initial experiments led to low conversions of aryl iodide and none of the desired product 3a (Table 1, entry 1). However, when 1 equivalent of a monochlorosilane additive such as TBSCl or TMSCl was added, low to moderate yields ( $24 \%$ and $55 \%$, respectively) of $\alpha$-aryl nitrile 3a were obtained (Table 1, entries 2-3). Di- and trichlorosilanes generally resulted in increased product formation, with the notable exception of $\mathrm{Si}\left({ }^{t} \mathrm{Bu}\right)_{2} \mathrm{Cl}_{2}$, which resulted in no detectable formation of $\mathbf{3 a}$ (Table 1, entries 47). Ultimately, when 3 equivalents of TMSCl were added in combination with $\mathrm{NiCl}_{2}$ bpy $(10 \mathrm{~mol} \%)$ as the pre-catalyst, and superstoichiometric $\mathrm{Zn}$ dust (8 equiv), 3a was obtained in $82 \%$ yield (Table 1, entry 8). ${ }^{11}$ Decreasing the amount of $\mathrm{Zn}$ to 2 equivalents resulted in reduced conversion to $\mathbf{3 a}$ (Table 1 , entry 9). The reaction is very rapid and occurs within 15 minutes at room temperature (vide infra), potentially signifying that rapid $\mathrm{Zn}$-mediated reduction of the Ni catalyst is essential for productive catalysis. Control reactions in the absence of $\mathrm{Ni}$, ligand or $\mathrm{Zn}$ resulted in no detectable product formation.

\section{Table 1. Effect of chlorosilane additive}

\begin{tabular}{|c|c|c|c|}
\hline$x$ & . & $\begin{array}{c}\mathrm{NiCl}_{2} \text { bpy }(10 \text { mol \%) } \\
\text { [Si]-Cl (1 equiv) } \\
\mathrm{Zn} \text { (8.0 equiv) }\end{array}$ & ${ }+X_{3}$ \\
\hline $\begin{array}{l}\text { NHP } \\
\text { 1a } \\
\text { (1.5 equiv) }\end{array}$ & (1 equiv) & $\begin{array}{c}\mathrm{DMA}(0.2 \mathrm{M}) \\
\text { r.t., } 1 \mathrm{~h}\end{array}$ & 3a OMe \\
\hline Entry & {$[\mathrm{Si}-\mathrm{Cl}]$} & yield $3 a(\%)^{a}$ & conv. $2(\%)^{a}$ \\
\hline 1 & None & 0 & 16 \\
\hline 2 & $\mathrm{TBSCl}$ & 24 & 53 \\
\hline 3 & TMSCl & 55 & 66 \\
\hline 4 & $\mathrm{SiCyMeCl}_{2}$ & 65 & 100 \\
\hline 5 & $\mathrm{Si}\left({ }^{\mathrm{i}} \mathrm{Pr}\right){ }_{2} \mathrm{Cl}_{2}$ & 74 & 100 \\
\hline 6 & $\mathrm{Si}\left({ }^{\mathrm{t}} \mathrm{Bu}\right)_{2} \mathrm{Cl}_{2}$ & 0 & 8 \\
\hline 7 & $\mathrm{SiPhCl}_{3}$ & 75 & 100 \\
\hline 8 & TMSCl (3 equiv) & 83 & 100 \\
\hline $9^{b}$ & TMSCl (3 equiv) & 53 & 83 \\
\hline
\end{tabular}

aCalibrated yields determined by GC-MS using dodecane as an internal standard. Reactions performed on a $0.1 \mathrm{mmol}$

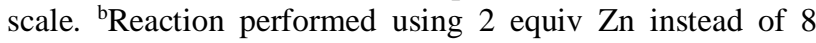
equiv.

After establishing the optimal reaction conditions, we set out to explore the scope of the reaction (Scheme 2). Notably, the reaction was selective for aryl iodides over other common cross-coupling partners such as aryl bromides (3t, 3v), aryl chlorides (3n), aryl tosylates (3b, 3k), and aryl pivalates (3w). Functionalization at the other reactive position of these substrates was never observed. ${ }^{12}$ Aryl iodides bearing basesensitive functional groups were also compatible, including a phenol (3c), sulfonamide (3d, 3u, 3y), benzyl alcohol (3g, 3t), and ketone (3h, 3z).

Notably, the unprotected alcohols in $\mathbf{3 c}, \mathbf{3 g}$, and $\mathbf{3 t}$ are silylated over the course of the reaction, and either the free alcohol $(\mathbf{3 c}, \mathbf{3 g})$, or the TMS-protected adduct $(\mathbf{3 t})$ can be isolated directly. ${ }^{13}$ Aryl iodides containing $N$-heterocycles $(3 \mathbf{i}, 3 \mathbf{s}, 3 \mathbf{u})$ were efficiently cross-coupled, as well as 5iodobenzothiophene $(\mathbf{3 j}, \mathbf{3 r})$. Tertiary amide substrate (3l), and secondary amide (3t) bearing a free $\mathrm{N}-\mathrm{H}$ were good coupling partners. Strongly electron-donating groups on the iodoarene are tolerated such as alkoxy $(\mathbf{3 a}, \mathbf{3 m}, \mathbf{3 q})$ and amino $(3 \mathbf{e}, 30)$ substituents. An ortho-substituted iodoarene gave the desired $\alpha$-aryl nitrile (3f) in $46 \%$ yield. Structurally complex aryl iodides, bearing a diverse range of functional groups (3su) were also suitable substrates in the reaction, which highlights the synthetic utility of this method within the context of late-stage diversification. In particular, $\beta$ hydroxyamide $\mathbf{3 t}$, is an intermediate towards an antihypercholesterolemic compound which features a selective $\mathrm{Ar}-\mathrm{I}$ over $\mathrm{Ar}-\mathrm{Br}$ functionalization en route towards those medicinal agents. ${ }^{14}$

Other NHP esters of functionalized cyanoacetic acids can also be used in this transformation. $\alpha$-Aryl nitriles with pendant alkyl chains (3v) or an allyl substituent (3w) were prepared in moderate to good yields from the corresponding NHP ester. Nitrile 3x was prepared in good yield from the NHP ester containing a free $\mathrm{N}-\mathrm{H}$ indole without requiring protecting group manipulations. ${ }^{15}$ Bulky isopropyl (3k-3r, 3t, 3u) and cyclohexyl (3y) substituted NHP esters were also suitable substrates for this transformation, as well as substrate $\mathbf{3 z}$ bearing a cyclopropane ring. ${ }^{16}$ 


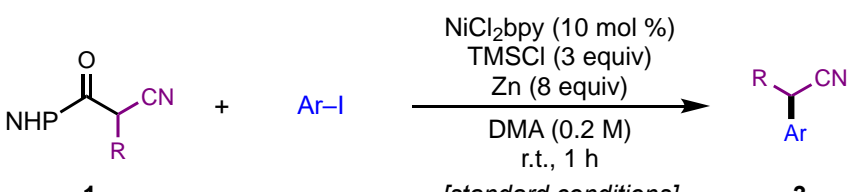

$$
\begin{aligned}
& 1 \\
& \text { (1.5 equiv) }
\end{aligned}
$$

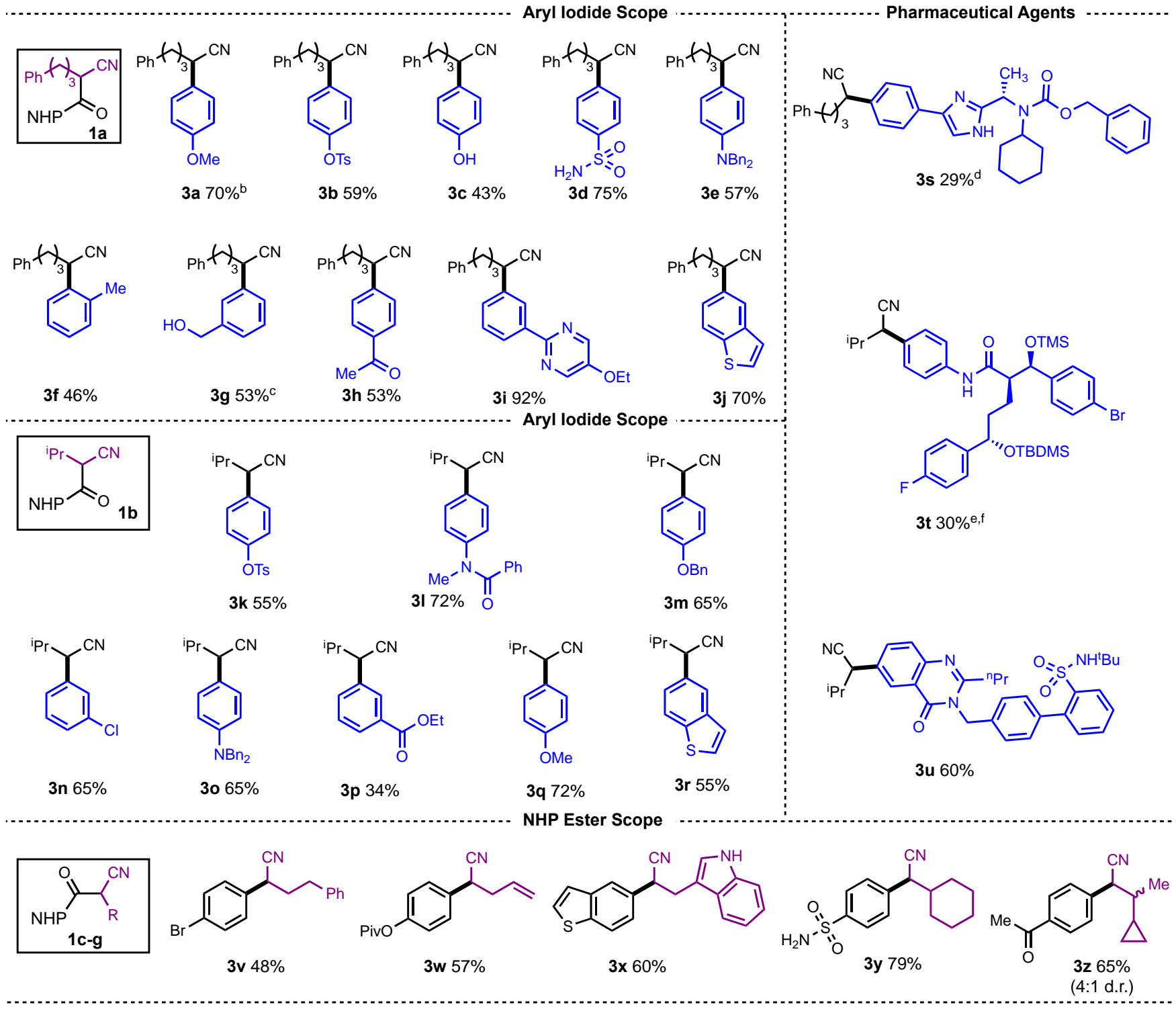

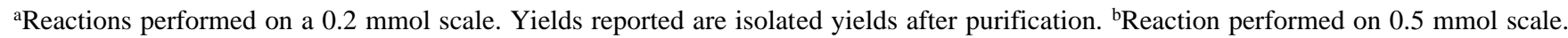
${ }^{\mathrm{c}}$ Reaction worked up with TBAF ( 2.5 equiv). ${ }^{\mathrm{d}}$ Reaction performed on $0.1 \mathrm{mmol}$ scale. ${ }^{\mathrm{e}} 4$ equiv $\mathrm{TMSCl}$ and 2 equiv $\mathbf{1 b}$ were used. ${ }^{\mathrm{f}} \mathrm{ArI}$ contained the free $\mathrm{OH}$ but was isolated as the OTMS adduct. 


\section{Scheme 3. Role of chlorosilane in product formation ${ }^{a}$}

a) $[\mathrm{Si}]-\mathrm{Cl}$ is a stoichiometic reagent

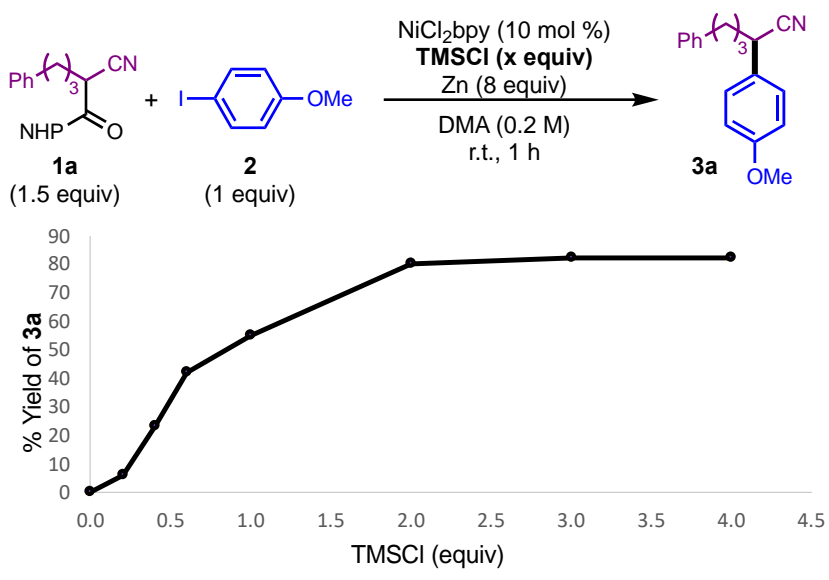

b) [Si]-Cl is required beyond the in-situ activation of $\mathrm{Zn}^{\mathrm{b}}$
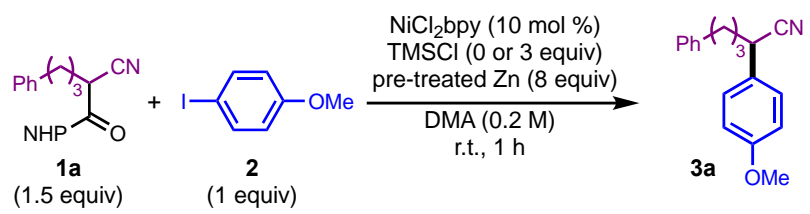

\begin{tabular}{cccc}
\hline Entry & TMSCl (equiv) & conv. 2 (\%) & yield 3a (\%) \\
\hline 1 & 0 & 11 & 0 \\
2 & 3 & 100 & 82
\end{tabular}

aReactions performed on $0.1 \mathrm{mmol}$ scale. Calibrated yields determined by GC-MS using dodecane as the internal standard. ${ }^{b}$ Pre-treated $\mathrm{Zn}$ was prepared by stirring with TMSCl (3 equiv) in DMA for 30 min. It was then filtered, washed with DMA, and dried in the glovebox before being added to the reaction under otherwise standard conditions.

Since the chlorosilane was an essential component to the reaction and, in its absence, no product was obtained (Table 1 , entry 1), we set out to investigate its role. Chlorosilanes have been used as additives for other reductive cross-coupling reactions. $^{9 \mathrm{~b}, 17}$ These reports typically suggest that the chlorosilane acts as an in-situ activator for the stoichiometric metal reductant $(\mathrm{Zn}$ or $\mathrm{Mn})$. While several reports use this reagent in catalytic amounts, some methods require stoichiometric (or near stoichiometric) quantities, which suggests a role beyond that of simply activating the reductant. ${ }^{9 \mathrm{~b}, 17 \mathrm{~d}, \mathrm{f}, \mathrm{g}, \mathrm{h}, \mathrm{i}}$ As the field of reductive cross-coupling continues to expand, detailed mechanistic understandings of the role of the components in these reactions will be essential to inspire future development.

Our initial mechanistic experiments demonstrated that TMSCl is necessary in stoichiometric amounts in the reaction (Scheme 3). As shown in Scheme 3a, we found that the yield of $\mathbf{3 a}$ increases with increasing amounts of TMSCl. To investigate whether the role of TMSCl was simply to act as an in-situ activator for $\mathrm{Zn}$, we pre-treated standard $\mathrm{Zn}$ dust ${ }^{18}$ with TMSCl (3 equiv) and subsequently used this as the stoichiometric reductant in the reaction (Scheme $3 b) .{ }^{19}$ Notably, in the absence of additional TMSCl, 3a was not observed which suggests that TMSCl plays a crucial role beyond solely acting as an activator for the metal reductant (Scheme 3b). ${ }^{20}$

\section{Scheme 4. Reaction kinetics ${ }^{\mathrm{a}}$}

a) Reaction kinetics

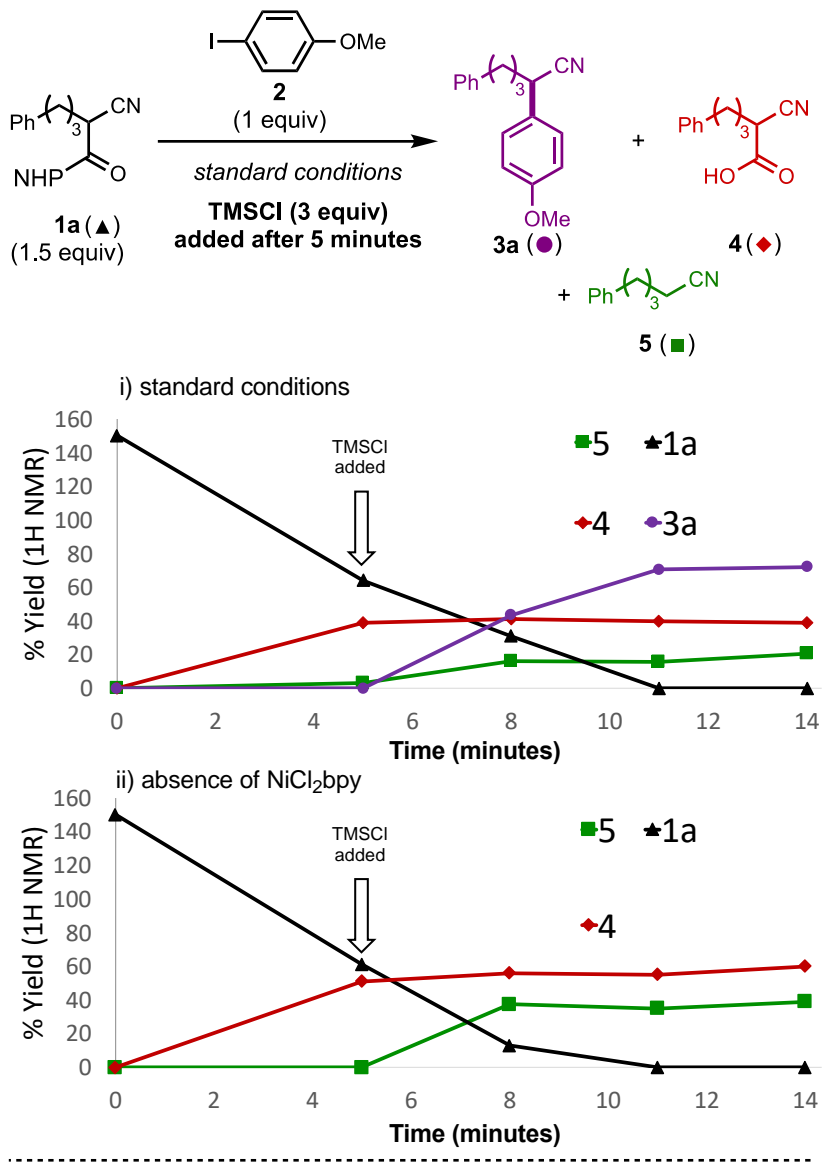

b) 4 is an off-cycle side product ${ }^{\mathrm{b}}$
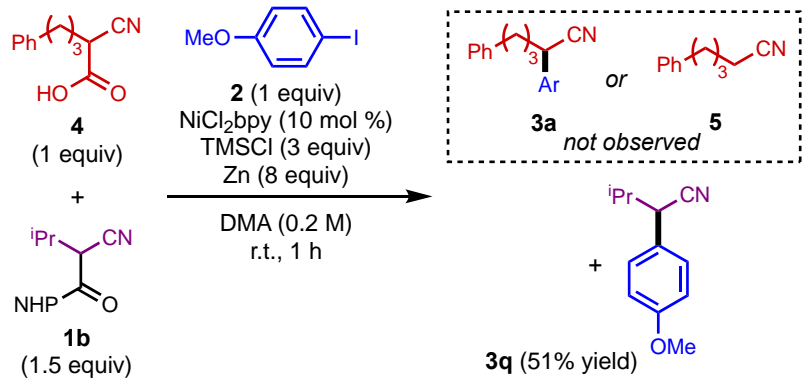

aReactions performed on a $0.3 \mathrm{mmol}$ scale. Yields determined by ${ }^{1} \mathrm{H}$ NMR using 1,3,5-trimethoxybenzene as an internal standard. ${ }^{b}$ Reaction performed on a $0.1 \mathrm{mmol}$ scale.

To further understand the role of $\mathrm{TMSCl}$, we studied the kinetics of the transformation, under slightly modified reaction conditions where $\mathrm{TMSCl}$ was added after 5 minutes (Scheme 4a, plot i). Interestingly, before the addition of TMSCl, we observed significant conversion of NHP ester 1a (black triangle) to the carboxylic acid $\mathbf{4}$ (red diamond), along with trace amounts (ca. 3\%) of decarboxylated side-product 5 (green square). ${ }^{21}$ Product 3a (purple circle) was not detected. Once TMSCl was added, however, we observed rapid formation of 3a, along with increased amounts of 
decarboxylated side-product 5 (ca. 20\%). It is important to note that carboxylic acid $\mathbf{4}$ is generated as a result of background hydrolysis of NHP ester 1a. ${ }^{22}$ When the same experiment was conducted in the absence of $\mathrm{NiCl}_{2}$ bpy (Scheme 4a, plot ii), we observed a similarly rapid decomposition of NHP ester 1a to carboxylic acid 4 . Decarboxylated side-product $\mathbf{5}$ was again not observed until TMSCl was added, at which point 1a was rapidly converted to $\mathbf{5}$ (ca. $40 \%$ in under 3 minutes). Importantly, when 1a is treated with TMSCl in DMA but in the absence of $\mathrm{Zn}, \mathbf{4}$ was observed and $\mathbf{5}$ was not detected. ${ }^{15}$ The most commonly accepted mechanism for NHP ester activation under $\mathrm{Ni}$ catalysis is through a single electron transfer (SET) from a low-valent $\mathrm{Ni}$ species $\left(\mathrm{Ni}^{(\mathrm{I})}\right.$ or $\left.\mathrm{Ni}^{(0)}\right)$ which leads to decarboxylation and liberation of the alkyl radical. ${ }^{8 \mathrm{a}, \mathrm{e}, 9 \mathrm{a}, \mathrm{d}, \mathrm{h}, 23}$ The observation that $\mathbf{5}$ is formed in appreciable amounts $(>5 \%)$ only after the addition of $\mathrm{TMSCl}$ suggests that reduction of the NHP ester 1a to the $\alpha$-cyano radical is mediated by a combination of chlorosilane and zinc.

To confirm that carboxylic acid $\mathbf{4}$ does not decarboxylate under the reaction conditions, we spiked $\mathbf{4}$ into a standard reaction between an alternative NHP ester substrate $\mathbf{1 b}$ and 4iodoanisole 2 (Scheme 4b). Decarboxylated side-product 5 and cross-coupled product $\mathbf{3 a}$ were not detected, while the expected arylated product $\mathbf{3 q}$ was still obtained in $51 \%{ }^{1} \mathrm{H}$ NMR yield. Since $\mathbf{5}$ is not generated from carboxylic acid $\mathbf{4}$, it is most likely being formed via direct conversion of the NHP ester $\mathbf{1 a}$ to the $\alpha$-cyano radical followed by subsequent reduction to the anion or HAT with a solvent molecule. ${ }^{24}$

\section{Scheme 5. Radical clock experiments}

a) Cyclopropane opening from $\mathrm{Zn}+\mathrm{TMSCl}$

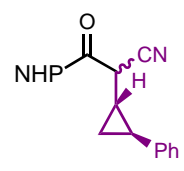

6

(1:1 d.r.)

b) Cyclopropane ring-opening is not observed without TMSCl

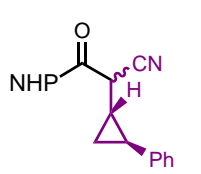

6

(1:1 d.r.)

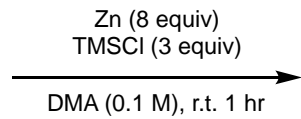

DMA $(0.1 \mathrm{M})$, r.t. $1 \mathrm{hr}$ bpy $(10 \mathrm{~mol} \%)$

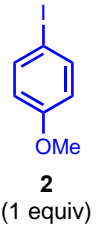
TMSCI ( 0 equiv) DMA (0.2 M), r.t. $1 \mathrm{hr}$

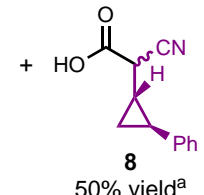

${ }^{a}$ Yield determined by ${ }^{1} \mathrm{H}$ NMR using 1,3,5trimethoxybenzene as an internal standard.

To further support the mechanistic hypothesis that TMSCl and $\mathrm{Zn}$ are responsible for $\alpha$-cyano radical formation from $\mathbf{1 a}$, we conducted a series of radical clock experiments. First, we prepared cyclopropane containing NHP ester $\mathbf{6}$ and subjected it to TMSCl and $\mathrm{Zn}$ in DMA (Scheme 5a). The expected ringopened acrylonitrile derivative 7 was isolated in $23 \%$ yield as a 7:3 E:Z mixture of isomers. ${ }^{25,26}$ For comparison, when 6 was submitted to the standard reaction conditions in the absence TMSCl, 7 was not detected and carboxylic acid $\mathbf{8}$ was observed as the major product (Scheme 5b). Importantly, no arylated products were detected during this reaction. Subjecting cyclopropane substrate $\mathbf{6}$ to the standard reaction conditions in the presence of TMSCl, $\mathrm{Zn}, \mathrm{Ni}$ and aryl iodide resulted in a complex mixture, and the expected $\delta$-arylated product could not be isolated.

\section{Scheme 6. Possible catalytic cycle}

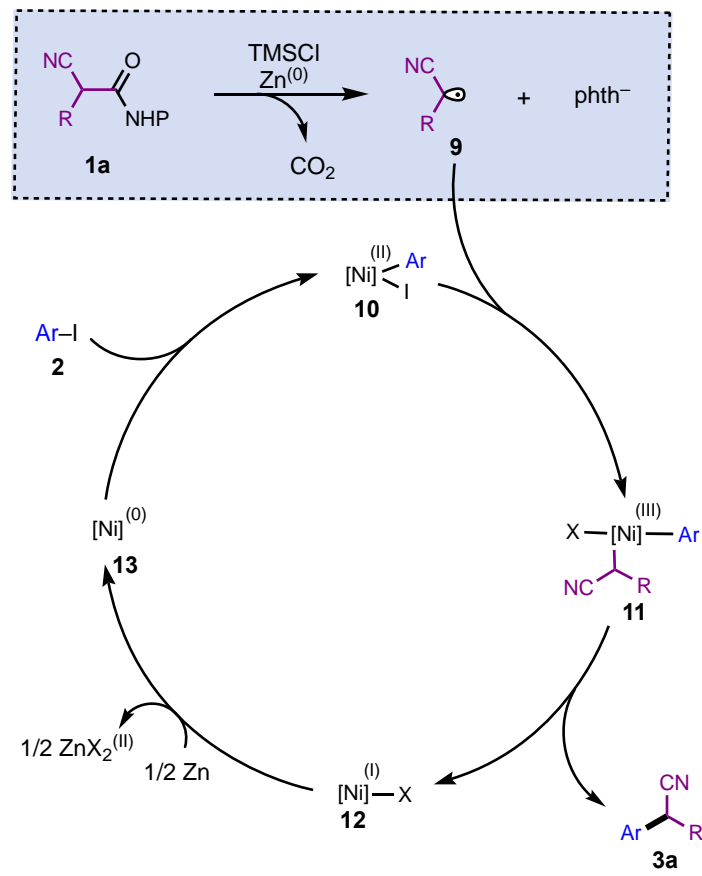

Based on these combined data and previous investigations, ${ }^{27,28}$ a plausible catalytic cycle is shown in Scheme 6. NHP ester 1a undergoes $\mathrm{TMSCl}$ mediated reduction by $\mathrm{Zn}$ to liberate the $\alpha$-cyano radical 9 via loss of $\mathrm{CO}_{2}$ and phthalimide anion $\left(\right.$ phth $\left.^{-}\right) .^{29}$ This radical is then captured by a Ni(II) oxidative addition complex $\mathbf{1 0}$ to generate $\mathrm{Ni}(\mathrm{III})$ intermediate 11, which upon reductive elimination furnishes the desired product 3a along with a $\mathrm{Ni}(\mathrm{I})$ species 12. Single electron reduction generates the $\mathrm{Ni}(0)$ complex 13 which could undergo an oxidative addition with iodoarene $\mathbf{2}$ thereby restarting the catalytic cycle. ${ }^{30}$ Since the consumption of NHP ester 1a to $\alpha$-cyano radical 9 is rapid and occurs in the absence of a nickel catalyst (Scheme 4), one explanation for the observed increase in yield when 8 equivalents of $\mathrm{Zn}$ are used (Table 1, entries 8 vs. 9) is that it enables rapid reduction of $\mathrm{Ni}(\mathrm{I})$ species $\mathbf{1 2}$ to a catalytically active $\mathrm{Ni}(0)$ species $\mathbf{1 3}$ and that this step is crucial in reducing the formation of side product 5 .

In conclusion, we have developed a $\mathrm{Ni}$-catalyzed reductive cross-coupling reaction of cyanoacetic acid-derived NHP esters and iodoarenes to yield valuable $\alpha$-aryl nitriles. The reaction conditions are mild and a broad scope of functional groups are tolerated as a result. A chlorosilane additive is necessary for the reaction to proceed and plays a crucial role in mediating NHP ester reduction and decarboxylation to a reactive $\alpha$-cyano radical intermediate. In its absence, decarboxylation only occurs in trace amount $(<5 \%)$ and cross-coupling is not observed. The prevalence of chlorosilanes as additives in RCC reactions renders this finding significant for mechanistic understanding and further developments in this field. Studies involving an enantioselective version of the reaction, along with utilizing 
other $\alpha$-functionalized NHP ester derivatives are currently underway in our group.

\section{ASSOCIATED CONTENT}

\section{Supporting Information}

The Supporting Information is available free of charge on the ACS Publications website.

Optimization details, experimental procedures, characterization of all products (file type, PDF)

NMR spectra (file type, $\mathrm{PDF}$ )

\section{AUTHOR INFORMATION}

\section{Corresponding Author}

* Sophie A. L. Rousseaux - Department of Chemistry, University of Toronto, Toronto, Ontario M5S 3H6, Canada; orcid.org/0000-0002-6505-5593

Email: sophie.rousseaux@utoronto.ca

\section{Authors}

Nicholas W. M. Michel - Department of Chemistry, University of Toronto, Toronto, Ontario M5S 3H6, Canada;

Racquel K. Edjoc - Department of Chemistry, University of Toronto, Toronto, Ontario M5S 3H6, Canada;

\footnotetext{
${ }^{1}$ For selected references on cross-electrophile coupling, see: a) Knappke, C. E. I.; Grupe, S.; Gärtner, D.; Corpet, M.; Gosmini, C.; Jacobi von Wangelin, A. Reductive Cross-Coupling Reactions between Two Electrophiles. Chem. Eur. J. 2014, 20, 6828-6842. b) Weix, D. J. Methods and Mechanisms for Cross-Electrophile Coupling of $\mathrm{Csp}^{2}$ Halides with Alkyl Electrophiles. Acc. Chem. Res. 2015, 48, 1767-1775. c) Goldfogel, M. J.; Huang, L.; Weix, D. J.; CrossElectrophile Coupling. In Nickel Catalysis in Organic Synthesis; Ogoshi, S., Ed.; Wiley-VCH: Weinheim, 2020; pp 183-222. d) Wang, X.; Dai, Y.; Gong, H.; Nickel-Catalyzed Reductive Couplings. Top. Curr. Chem. 2016, 374, 43. e) Everson, D. A.; Weix, D. J.; CrossElectrophile Coupling: Principals of Reactivity and Selectivity. J. Org. Chem. 2014, 79, 4793-4798.

${ }^{2}$ For the importance of the nitrile functional group, see: (a) Fleming, F. F.; Yao, L.; Ravikumar, P. C.; Funk, L.; Shook, B. C. NitrileContaining Pharmaceuticals: Efficacious Roles of the Nitrile Pharmacophore. J. Med. Chem. 2010, 53, 7902-7917. (b) Fleming, F. F. Nitrile-containing Natural Products. Nat. Prod. Rep. 1999, 16, 597-606.

${ }^{3}$ For reviews, see: (a) Culkin, D. A.; Hartwig, J. F. Palladium Catalyzed $\alpha$-Arylation of Carbonyl Compounds and Nitriles. Acc. Chem. Res. 2003, 36, 234-245. (b) Johansson, C. C. C.; Colacot, T. J. Metal-Catalyzed a-Arylation of Carbonyl and Related Molecules: Novel Trends in $\mathrm{C}-\mathrm{C}$ Bond Formation by $\mathrm{C}-\mathrm{H}$ Bond Functionalization. Angew. Chem., Int. Ed. 2010, 49, 676-707.

${ }^{4}$ For arylation see: a) Shang, R.; Ji, D-S.; Chu, L.; Fu, Y.; Liu, L. Synthesis of $\alpha$-Aryl Nitriles through Palladium-Catalyzed Decarboxylative Coupling of Cyanoacetate Salts with Aryl Halides and Triflates. Angew. Chem. Int. Ed. 2011, 50, 4470-4474. For intramolecular allylation see: b) Recio III, A.; Tunge, J. A.
}

Emmanuel Fagbola - Department of Chemistry, University of Toronto, Toronto, Ontario M5S 3H6, Canada;

Jonathan M. E. Hughes - Department of Process Research and Development, Merck \& Co. Inc., Rahway New Jersey 07065, United States

Louis-Charles Campeau - Department of Process Research and Development, Merck \& Co. Inc., Rahway New Jersey 07065, United States

All authors have given approval to the final version of the manuscript.

\section{ACKNOWLEDGMENT}

We thank NSERC (Discovery Grants and Canada Research Chair programs), the Canada Foundation for Innovation (Project No. 35261), the Ontario Research Fund, Kennarshore Inc., and the University of Toronto for generous financial support of this work. We also acknowledge the Canada Foundation for Innovation (Project No. 19119) and the Ontario Research Fund for funding the Centre for Spectroscopic Investigation of Complex Organic Molecules and Polymers. N.W.M.M thanks NSERC and the province of Ontario for graduate scholarships (CGS-D, OGS). E.F thanks NSERC for an undergraduate summer research award (USRA). Dr. Patrick Fier and Dr. Sue Zultanski are thanked for critical reading of the manuscript. Dr. L. Reginald Mills and John Monteith are thanked for insightful discussions. Dr. Karl Demmans, and Dr. Darcy Burns are thanked for assistance with NMR.

\section{REFERENCES}

Regiospecific Decarboxylative Allylation of Nitriles. Org. Lett. 2009, 11,5630 .

5 For traditional cross-coupling arylation reactions using electrophilic nitrile reagents, see: a) Choi, Junwon, Fu. C. G. Catalytic Asymmetric Synthesis of Secondary Nitriles via Stereoconvergent Negishi Arylations and Alkenylations of Racemic $\alpha$-Bromonitriles. $J$. Am. Chem. Soc. 2012, 134, 9102-9105. b). For a Pd catalyzed method, see: b) He, A.; Falck, J. R. Stereospecific Suzuki Cross-Coupling of Alkyl $\alpha$-Cyanohydrin Triflates. J. Am. Chem. Soc. 2010, 132, 25242525.

${ }^{6}$ For reductive cross-coupling reactions using electrophilic nitrile reagents, see: Kadunce, N. T.; Reisman, S. E. Nickel Catalyzed Asymmetric Reductive Cross-Coupling between Heteroaryl Iodides and $\alpha$-Chloronitriles. J. Am. Chem. Soc. 2015, 137, 10480- 10483.

${ }^{7}$ For reductive cross-coupling reactions featuring a $\alpha$-substituted EWG in related systems, see: Guan, H.; Zhang, Q.; Walsh, P. J.; Mao, J. Nickel/Photoredox-Catalyzed Asymmetric Reductive CrossCoupling of Racemic $\alpha$-Chloro Esters with Aryl Iodides. Angew. Chem. Int. Ed. 2020, 59, 5172-5177.

${ }^{8}$ For other Ni catalyzed functionalizations of NHP esters see: a) Qin, T.; Cornella, J.; Li, C.; Malins, L. R.; Edwards, J. T.; Kawamura, S.; Maxwell, B. D.; Eastgate, M. D. Baran, P. S. A general alkyl-alkyl cross-coupling enabled by redox-active esters and alkylzinc reagents. Science 2016, 352, 801-805. b) Tortajada, A.; Duan, Y.; Sahoo, B.; Cong, F.; Toupalas, G.; Sallustrau, A.; Loreau, O.; Audisio, D.; Martin, R. Catalytic Decarboxylation/Carboxylation Platform for Accessing Isotopically Labeled Carboxylic Acids. ACS Catal. 2019, 9, 58975901. c) Chen, T-G.; Zhang, H.; Mykhailiuk, P. K.; Merchant, R. R.; Smith, C. A.; Quin, T.; Baran, P. S. Quaternary Centers by NickelCatalyzed Cross-Coupling of Tertiary Carboxylic Acids and (Hetero)Aryl Zinc Reagents. Angew. Chem. Int. Ed. 2019, 58, 2454- 
2458. d) Cornella, J.; Edwards J. T.; Quin, T.; Kawamura, S.; Wang, J.; Pan, C-M.; Gianatassio, R.; Schmidt, M.; Eastgate, M. D.; Baran, P. S. Practical Ni-Catalyzed Aryl-Alkyl Cross-Coupling of Secondary Redox-Active Esters. J. Am. Chem. Soc. 2016, 138, 2174-2177. e) Ni. S.; Padial, N. M.; Kingston, C.; Vantourout, J. C.; Schmitt, D. C.; Edwards, J. T.; Kruszyk, M. M.; Merchant, R. R.; Mykhailiuk, P. K.; Sanchez, B. B.; Yang, S.; Perry, M. A.; Gallego, G. M.; Mousseau, J. J.; Collins, M. R.; Cherney, R. J.; Lebed, P. S.; Chen, J. S.; Qin, T.; Baran, P. S. A Radical Approach to Anionic Chemistry: Synthesis of Ketones, Alcohols, and Amines. J. Am. Chem. Soc. 2019, 141, 67266739. f) Edwards, J. T.; Merchant, R. R.; McClymont, K. S.; Knouse, K. W.; Qin, T.; Malins, L. R.; Vokits, B.; Shaw, S. A.; Bao, D-H.; Wei, F-L.; Zhou, T.; Eastgate, M. D.; Baran, P. S. Decarboxylative alkenylation. Nature 2017, 545, 213-219. g) Qin, T.; Malins, L. R.; Edwards, J. T.; Merchant, R. R.; Novak, A. J. E.; Zhong, Z.; Mills, R. B.; Yan, M.; Yuan, C.; Eastgate, M. D.; Baran, P. S. Nickel-Catalyzed Barton Decarboxylation and Giese Reactions: A Practical Take on Classic Transforms. Angew. Chem. Int. Ed. 2017, 56, 260-265. h) Chen. T-G.; Barton, L. M.; Lin, Y.; Tsien, J.; Kossler, D.; Bastida, I.; Asai, S.; Bi, C.; Che, J. S.; Shan, M.; Fang, H.; Fang, F. G.; Choi, Hw.; Hawkins, L.; Qin, T.; Baran, P. S. Building C $\left(s p^{3}\right)$-rich complexity by combining cycloaddition and $\mathrm{C}-\mathrm{C}$ cross-coupling reactions. Nature, 2018, 560, 350-354. i) Smith, J. M.; Qin, T.; Merchant, R. R.; Edwards, J. T.; Malins, L, R.; Liu, Z.; Che, G.; Shen, Z.; Shaw, S. A.; Eastgate, M. D.; Baran, P. S. Decarboxylative Alkynalation. Angew. Chem. Int. Ed. 2017, 56, 11906-11901. j) Wang, J.; Qin, T.; Chen, T-G.; Wimmer, L.; Edwards, J. T.; Cornella, J.; Vokits, B.; Shaw, S. A.;1 Baran, P. S. Nickel-Catalyzed Cross-Coupling of Redox-Active Esters with Boronic Acids. Angew. Chem. Int. Ed. 2016, 55, 9676-9679.

${ }^{9}$ For reductive cross-coupling reactions using NHP esters and a stoichiometric reductant, see: a) Huihui, K. M. M.; Caputo, J. A.; Melchor, Z.; Olivares, A. M.; Spiewak, A. M.; Johnson, K. A.; DiBenedetto, T. A.; Kim, S.; Ackerman, L. K. G.; Weix, D. J. Decarboxylative Cross-Electrophile Coupling of $\mathrm{N}$ Hydroxyphthalimide Esters with Aryl Iodides. J. Am. Chem. Soc. 2016, 138, 5016-5019. b) Suzuki, N.; Hofstra, J. L.; Poremba, K. E.; Reisman, S. E. Nickel-Catalyzed Enantioselective Cross-Coupling of $N$-Hydroxyphthalimide Esters with Vinyl Bromides. Org. Lett. 2017, 19, 2150-2153. c) Lu, X.; Wang, X-X.; Gong, T-J.; Pi, J-J.; He, S-J.; Fu, Y. Nickel-Catalyzed Allylic Defluorinative Alkylation of Trifluoromethyl Alkenes with Reductive Decarboxylation of RedoxActive Esters. Chem. Sci. 2019, 10, 809-814. d) Huang, L.; Olivares, A. M.; Weix, D. J. Reductive Decarboxylative Alkynylation of NHydroxyphthalimide Esters with Bromoalkynes. Angew. Chem. 2017, 129, 12063-12067. e) Jin, Y.; Yang, H.; Wang, C. Nickel-Catalyzed Reductive Arylalkylation via a Migratory Insertion/Decarboxylative Cross-Coupling Cascade. Org. Lett. 2019, 21, 7602-7608. f) Watanabe, E.; Chen, Y.; May, O.; Ley, S. V. A Practical Method for Continuous Production of $\mathrm{Sp}^{3}$-Rich Compounds from (Hetero)Aryl Halides and Redox-Active Esters. Chem. Eur. J. 2020, 26, 186-191. g) Wang, J.; Cary, B. P.; Beyer, P. D.; Gellman, S. H.; Weix, D. J. Ketones from Nickel-Catalyzed Decarboxylative, Non-Symmetric CrossElectrophile Coupling of Carboxylic Acid Esters. Angew. Chem. 2019, 131, 12209-12213. h) Yang, T.; Jiang, Y.; Luo, Y.;Lim, J. J. H.; Lan, Y.; Koh, M. J. Chemoselective Union of Olefins, Organohalides, and Redox-Active Esters Enables Regioselective Alkene Dialkylation. $J$. Am. Chem. Soc. 2020, 142, 21410-21419. For reductive cross-coupling reactions using NHP esters under electrochemical reduction, see: i) Koyanagi, T.; Herath, A.; Cong, A.; Ratnikov, M.; Valiere, A.; Chang, J. Molteni, V.; Loren, J. One-Pot Electrochemical Nickel-Catalyzed Decarboxylative $\mathrm{Sp}^{2}-\mathrm{Sp}^{3}$ Cross-Coupling. Org. Lett. 2019, 21, 816820. j) Li, H.; Breen, C. P.; Seo, H.; Jamison, T. F.; Fang, Y-Q.; Bio, M. M. Ni-Catalyzed Electrochemical Decarboxylative $\mathrm{C}-\mathrm{C}$ Couplings in Batch and Continuous Flow. Org. Lett. 2018, 20, 1338-1341. For leading reviews, see: k) Muraka, S. Adv. Synth. Catal. 2018, 260, 17351753. 1) Niu, P.; Li, J.; Zhang, Y.; Huo, C. Eur. J. Org. Chem. 2020, 5801-5814. m) Chen, H.; Lui, Y. A.; Liao, X. Synthesis, 2021, 53, 129. m) Parida, S. K.; Mandal, T.; Das, S.; Hota, S. K.; Sarkar, S. D.; Muraka, S. Single Electron Transfer-Induced Redox Processes Involving N-(Acyloxy)phthalimides. ACS. Catal. 2021, 11, 16401683.

${ }^{10} \mathrm{RCC}$ reactions of NHP esters bearing an $\alpha$-amino (See refs $9 \mathrm{c}$, d, f, g, i) or an $\alpha$-alkoxy (See refs 9b, c, g, h, i, j) substituent have been documented. However, examples of substrates bearing an electrondeficient group in the $\alpha$-position are notably absent.

${ }^{11}$ See the supporting information for more detailed optimization.

12 Rosen, B. M.; Quasdorf, K. W.; Wilson, D. A.; Zhang, N.; Resmerita, A-M.; Garg, N. K.; Percec, V. Nickel-Catalyzed CrossCouplings Involving Carbon-Oxygen Bonds. Chem. Rev. 2011, 111, 1346-1416.

${ }^{13}$ Phenolic substrate $\mathbf{3 c}$ was isolated as the free $-\mathrm{OH}$ due to the TMS group falling off during chromatography. Product $\mathbf{3 g}$ was desilylated by addition of TBAF (1.0 M in THF, 2.5 equiv). 3t was directly isolated as the OTMS adduct. See SI for details.

14 a) Limanto, J.; Krska, S. W.; Dorner, B. T.; Vazquez, E.; Yoshikawa, N.; Tan, L. Dynamic Kinetic Resolution: Asymmetric Transfer Hydrogenation of $\alpha$-Alkyl-Substituted $\beta$-Ketoamides. Org. Lett. 2010, 12, 512, 515. b) Limanto, J.; Lushi, T.; Dreher, S. D.; Dorner, B. T.; Naoki, Y.; Krska, S. W. Process for Preparing an AntiHypercholesterolemic Compound. U.S. Patent WO 2009054887. April $30,2009$.

${ }^{15}$ See supporting information for more details.

${ }^{16}$ See supporting information for the complete scope of the reaction including substrates that were less tolerated.

${ }^{17}$ For reductive cross-coupling reactions that use a chlorosilane additive with a Mn reductant, see: a) Nimmagadda, S. K.; Korapati, S.; Dasgupta, D.; Malik, N. A.; Vinodini, A.; Gangu, A. S.; Kalidindi, S.; Maity, P.; Bondigela, S. S.; Venu, A.; Gallagher, W. P.; Aytar, S.; González-Bobes, F.; Vaidyanathan, R. Development and Execution of an Ni(II)-Catalyzed Reductive Cross-Coupling of Substituted 2Chloropyridine and Ethyl 3-Chloropropanoate. Org. Process Res. Dev. 2020, 24, 1141-1148. b) Kadunce, N. T.; Reisman, S. E. Nickel Catalyzed Asymmetric Reductive Cross-Coupling between Heteroaryl Iodides and $\alpha$-Chloronitriles. J. Am. Chem. Soc. 2015, 137, 1048010483. c) Johnson, K. A.; Biswas, S.; Weix, D. J. Cross-Electrophile Coupling of Vinyl Halides with Alkyl Halides. Chem. Eur. J. 2016, 22, 7399 - 7402; d) Shimkin, K. W.; Montgomery, J. Synthesis of Tetrasubstituted Alkenes by Tandem Metallacycle Formation/CrossElectrophile Coupling. J. Am. Chem. Soc. 2018, 140, 7074-7078. e) Tu, H-Y.; Wang, F.; Huo, L.; Li, Y.; Zhu, S.; Xhao, X.; Li, H.; Qing, F-L.; Chu, L. Enantioselective Three-Component Fluoroalkylarylation of Unactivated Olefins through Nickel-Catalyzed Cross-Electrophile Coupling. J. Am. Chem. Soc. 2020, 142, 9604-9611. f) Poremba, K. E.; Kadunce, N. T.; Suzuki, N.; Cherney, A. H.; Reisman, S. E. NickelCatalyzed Asymmetric Reductive Cross-Coupling To Access 1,1Diarylalkanes. J. Am. Chem. Soc. 2017, 139, 5684-5687. For reductive cross-coupling using a chlorosilane with a $\mathrm{Zn}$ reductant, see: g) Lin, T.; Mi, J.; Song, L.; Gan, J.; Luo, P.; Mao, J.; Walsh, P. J. NickelCatalyzed Desymmetrizing Cross-Electrophile Coupling of Cyclic Meso-Anhydrides. Org. Lett. 2018, 20, 1191-1194. h) Lin, Z.; Lan, Y.; Wang, C. Synthesis of gem-Difluoroalkenes via NickelCatalyzed Reductive $\mathrm{C}-\mathrm{F}$ and $\mathrm{C}-\mathrm{O}$ Bond Cleavage. ACS. Catal. 2019 9, 775-780. i) Chenniappan, V. K.; Peck, D.; Rahaim, R. Nickel catalyzed deoxygenative cross-coupling of benzyl alcohols with arylbromides. Tetrahedron Letters, 2020, 61, 151729. j) Arendt, K. M.; Doyle, A. G. Dialkyl Ether Formation by Nickal-Catalyzed CrossCoupling of Acetals and Aryl Iodides. Angew. Chem. Int. Ed. 2015, 54, 9876-9880. h) Chandrachud, P. P.; Wojtas, L.; Lopchuk, J. M. Decarboxylative Amination: Diazirines as Single and Double Electrophilic Nitrogen Transfer Reagents. J. Am. Chem. Soc. 2020, 142, 21743-21750. i) Ze-Peng, Y.; Freas, D. J.; Fu, G. C. The Asymmetric Synthesis of Amines via Nickel-Catalyzed Enantioconvergent Substitution Reactions. J. Am. Chem. Soc. ASAP, doi: 10.1021/jacs.0c13034. For reductive cross-coupling using organic reductants, see Ref $9 b$

${ }^{18}$ The standard $\mathrm{Zn}$ dust used in the reaction was activated with dilute HCl; Fieser, L. F.; Fieser, M. Fieser and Fieser's Reagents for Organic Synthesis, Volume 1; John Wiley and Sons, Inc.: New York, 1967.

${ }^{19} \mathrm{Pre}$-treated $\mathrm{Zn}$ was prepared by stirring with 3 equiv of $\mathrm{TMSCl}$ in DMA for 30 min before being filtered, washed (DMA), and dried in the glovebox. It was then added to the reaction under otherwise standard conditions.

20 Without additional TMSCl (Scheme 2b, entry 1) $11 \%$ of iodoarene 2 was consumed (using $10 \mathrm{~mol} \% \mathrm{Ni}$ catalyst), which 
suggests that while the Ni catalyst is active, in the absence of TMSCl subsequent steps in the catalytic cycle are not operative.

21 The unaccounted-for mass balance of NHP ester 1a is likely due to loss of carboxylic acid $\mathbf{4}$ to the aqueous layer during the work up of the aliquots.

22 During control reactions in the absence of TMSCl, Nhydroxyphthalimide is formed alongside carboxylic acid $\mathbf{4}$ and phthalimide is not detected. This suggests that carboxylic acid $\mathbf{4}$ arises via hydrolysis and not through a reductive mechanism.

${ }^{23}$ For reactions where $\mathrm{Zn}$ or $\mathrm{Mn}$ are able to generate an alkyl radical from NHP esters, see: a) Yu, L.; Tang, M-L.; Si, C-M.; Meng, Z.; Liang, Y.1 Han, J.; Sun, X. Zinc-Mediated Decarboxylative Alkylation of Gem-difluroalkenes. Org. Lett. 2018, 20, 4579-4583. b) Jiang, WT.; Yang, S.; Xu, M-Y.; Xie, X-Y.; Xiao, B. Zn-Mediated Decarboxylative Carbagermatranation of Aliphatic NHydroxyphthalimide Esters: Evidence for an Alkylzinc Intermediate. Chem. Sci. 2020, 11, 488-493. c) Li, Z.; Wang, K-F.; Zhao, X.; Ti, H.; Liu, X-G.; Wang, H. Manganese-Mediated Reductive Functionalization of Activated Aliphatic Acids and Primary Amines. Nature Communications, 2020, 11, 5036.

24 a) M'Halla, F.; Pinson, J.; Savéant, J. M. The Solvent as H-Atom Donor in Organic Electrochemical Reactions. Reduction of Aromatic Halides. J. Am. Chem. Soc. 1980, 102, 4120-4127. b) Bajo, S.; Laidlaw, G.; Kennedy, A. R.; Sproules, S.; Nelson, D. J. Oxidative Addition of Aryl Electrophiles to a Prototypical Nickel(0) Complex: Mechanism and Structure/Reactivity Relationships. Organometallics 2017, 36, 1662-1672. c) Keller, C. L.; Dalessandro, J. D.; Hotz, R. P.; Pinhas, A. R. Reactions in Water: Alkyl Nitrile Coupling Reactions Using Fenton's Reagent. J. Org. Chem. 2008, 73, 3616-3618.

25 The remainder of the mass balance in this reaction is very likely carboxylic acid 8. An exact yield of $\mathbf{8}$ was not obtained as the compound was partially lost when the reaction mixture was filtered over Si upon workup.
${ }^{26}$ Nonhebel, D. C. The chemistry of cyclopropylmethyl and related radicals. Chem. Soc. Rev. 1993, 22, 347-359

27 a) Biswas, S.; Weix, D. J. Mechanism and Selectivity in NickelCatalyzed Cross-Electrophile Coupling of Aryl Halides with Alkyl Halides. J. Am. Chem. Soc. 2013, 135, 16192-12197. b) Poremba, K. E.; Dibrell, S. E.; Reisman, S. E. Nickel-Catalyzed Enantioselective Reductive Cross-Coupling Reactions. ACS. Catal. 2020, 10, 8237 8246. c) Ren, Q.; Jiang, F.; Gong, H. DFT study of the single electron transfer mechanisms in Ni-Catalyzed reductive cross-coupling of aryl bromide and alkyl bromide. J. Organomet. Chem. 2014, 770, 130-135. e) Lin, Q.; Diao, T. Mechanism of Ni-Catalyzed Reductive 1,2Dicarbofunctionalization of Alkenes. J. Am. Chem. Soc. 2019, 141, 17937-17948.

${ }^{28}$ For selected examples of radical chain mechanism in reductive cross-coupling reactions, see: a) Ackerman, L. K. G.; Anka-Lufford, L. L.; Naodovic, M.; Weix, D. J. Cobalt co-catalysis for cross-electrophile coupling: diarylmethanes from benzyl mesylates and aryl halides. Chem. Sci. 2015, 6, 1115-1119. b) Zhao, Y.; Weix, D. J. NickelCatalyzed Regiodivergent Opening of Epoxides with Aryl Halides: CoCatalysis Controls Regioselectivity. J. Am. Chem. Soc. 2014, 136, 4851. c) Zhang, P.; Le, C.; MacMillan, D. W. C. Silyl Radical Activation of Alkyl Halides in Metallaphotoredox Catalysis: A Unique Pathway for Cross-Electrophile Coupling. J. Am. Chem. Soc. 2016, 138, 80848087.

29 The details of the interaction between NHP ester 1a and TMSCl leading to decarboxylation are not yet fully understood. Experimental and computational studies are underway to clarify this interaction.

30 Another plausible catalytic cycle involves radical capture by a $\mathrm{Ni}(0)$ species followed by oxidative addition of the resulting $\mathrm{Ni}(\mathrm{I})$ complex with the iodoarene. Both catalytic cycles are consistent with the data. 